\title{
Random Logistic Maps II, the critical case
}

\author{
K. B. ATHREYA* AND H.-J. SCHUH** \\ Cornell University and Johannes Gutenberg-Universität, Mainz
}

\section{Abstract:}

Let $\left(X_{n}\right)_{0}^{\infty}$ be a Markov chain with state space $S=[0,1]$ generated by the iteration of i.i.d. random logistic maps, i.e. $X_{n+1}=C_{n+1} X_{n}\left(1-X_{n}\right), n \geq 0$, where $\left(C_{n}\right)_{1}^{\infty}$ are i.i.d. random variables with values in $[0,4]$ and independent of $X_{0}$. In the critical case, i.e. when $E\left(\log C_{1}\right)=0$, Athreya and Dai [2] have shown that $X_{n} \stackrel{P}{\longrightarrow} 0$. In this paper it is shown that if $P\left(C_{1}=1\right)<1$ and $E\left(\log C_{1}\right)=0$ then

i) $X_{n}$ does not go to zero with probability one (w.p.1) and in fact, there exists a $0<$ $\beta<1$ and a countable set $\triangle \subset(0,1)$ such that for all $x \in(0,1) \backslash \triangle, P_{x}\left(X_{n} \geq \beta\right.$ for infinitely many $n \geq 1)=1$, where $P_{x}$ stands for the probability distribution of $\left(X_{n}\right)_{0}^{\infty}$ with $X_{0}=x$ w.p.1.

ii) If $\gamma$ is the supremum of the support of the distribution of $C_{1}$, then for all $x \in(0,1) \backslash \triangle$

a) $P_{x}\left(\varlimsup_{n} X_{n}=1-\frac{1}{\gamma}\right)=1$ for $1 \leq \gamma \leq 2$

b) $P_{x}\left(\varlimsup_{n} X_{n} \geq 1-\frac{1}{\gamma}\right)=1$ for $2 \leq \gamma \leq 4$

c) $P_{x}\left(\varlimsup_{n} X_{n}=\frac{\gamma}{4}\right)=1$ for $2 \leq \gamma \leq 4$ under some additional smoothness condition on the distribution of $C_{1}$.

iii) The empirical distribution $V_{n}(.) \equiv \frac{1}{n} \sum_{0}^{n-1} I\left(X_{j} \in\right.$.) converges weakly to $\delta_{0}$, the delta measure at 0, w.p.1 for any initial distribution of $X_{0}$.

*Research supported by NSF.

*Research done during a visit to the School of ORIE, Cornell University. 
Keywords: Random logistic maps, critical case, convergence in probability but not w.p.1, empirical distribution.

AMS (1991) subject classification: Primary 60J05,92D25 Secondary 60F05.

$\begin{array}{ll}\text { K. B. Athreya } & \text { H.-J. Schuh } \\ \text { School of ORIE } & \text { Fachbereich 17, Mathematik, } \\ \text { Rhodes Hall } & \text { Johannes Gutenberg-Universität, } \\ \text { Cornell University } & \text { D-55099 Mainz, } \\ \text { Ithaca, NY 14853 } & \text { Germany } \\ \text { email: athrerya@orie.cornell.edu } & \text { email: schuh@mat.mathematik.uni-mainz.de }\end{array}$




\section{Introduction}

The logistic map

$$
g_{c}(x) \equiv c x(1-x), 0 \leq x \leq 1, \text { with } 0 \leq c \leq 4
$$

is of great relevance in ecology, especially in predator-prey population dynamics ( $R$. M. May [9]). It is also an important special case in the theory of discrete time dynamical systems (Devaney [7]). The study of the case when the growth parameter $c$ changes randomly was initiated by Bhattacharya and Rao [4]. Over the last few years contributions were made by Bhattacharya and Majumdar [3], Bhattacharya and Waymire [5], Athreya and Dai [2] and Dai [6] among others. For a succinct review of these contributions see the survey by Athreya and Bhattacharya [1].

Athreya and Dai [2] introduced the following trichotomy for the study of the asymptotic behavior of the random dynamical system $\left(X_{n}\right)_{0}^{\infty}$ generated by the iteration of i.i.d. random maps

$$
X_{n+1}=g_{c_{n+1}}\left(X_{n}\right)=C_{n+1} X_{n}\left(1-X_{n}\right)
$$

where $\left(C_{n}\right)_{1}^{\infty}$ are i.i.d. r.v.'s with values in $(0,4]$ and independent of $X_{0}$ with values in $[0,1]$. Clearly $\left(X_{n}\right)_{0}^{\infty}$ is a Markov chain with state space $[0,1]$. They define $\left(X_{n}\right)_{0}^{\infty}$ to be subcritical, critical or supercritical according as

$$
E\left(\log C_{1}\right)<0,=0 \text { or }>0
$$

They showed that if $E\left(\log C_{1}\right)<0$, then $X_{n} \rightarrow 0$ w.p.1 at a geometric rate, and if $E\left(\log C_{1}\right)=0$, then $X_{n} \rightarrow 0$ in probability, while if $E\left(\log C_{1}\right)>0$ and $E(\mid \log (4-$ $\left.\left.C_{1}\right) \mid\right)<\infty$, then there is a stationary probability distribution $\pi$ on the open interval $(0,1)$, and further, $X_{n}$ converges in distribution to such a $\pi$ under some further con- 
ditions on the distribution of $C_{1}$.

In the deterministic critical case, when $C_{1} \equiv 1$ w.p.1, the sequence $X_{n}$ does go to zero for any initial value (see Devaney [7]). In fact,

$$
\frac{1}{2 n} \leq X_{n} \leq \frac{1}{n}, \text { if } X_{2} \equiv \frac{1}{2}
$$

as can be seen by a straightforward induction. But when $C_{1}$ is random and $E\left(\log C_{1}\right)=$ $0, X_{n} \rightarrow 0$ in probability as shown by Athreya and Dai [2]. A natural question is whether this can be improved to $X_{n} \rightarrow 0$ w.p.1. The main result of the present paper is that the answer to this question is an emphatic no.

It is shown in Theorem 1 that there is a level $\beta, 0<\beta<1$ and a countable set $\triangle \subset(0,1)$ such that $P_{x}\left(X_{n} \geq \beta\right.$ for some $\left.n \geq 1\right)=1$ for all $x \in(0,1)-\triangle$ and hence by the Markov property, $P_{x}\left(X_{n} \geq \beta\right.$ for infinitely many $\left.n \geq 1\right)=1$ for all $x \in(0,1) \backslash \triangle$.

Here $P_{x}$ stands for the probability distribution of $\left(X_{n}\right)_{0}^{\infty}$ when $X_{0}=x$ w.p. 1 . The proof of Theorem 1 is based on a martingale method. As a sequel to Theorem 1 we also show that the sequence $\left(X_{n}\right)_{0}^{\infty}$ can reach higher levels as well. In fact, we show in Theorem 2 that if $\gamma$ is the supremum of the support of $C_{1}$, then for any $P_{x}, x \in(0,1) \backslash \triangle$, w.p.1:

$$
\varlimsup_{n} X_{n}=1-\frac{1}{\gamma}, \text { if } 1 \leq \gamma \leq 2 \text { and } \varlimsup_{n} X_{n} \geq 1-\frac{1}{\gamma}, \text { if } 2 \leq \gamma \leq 4
$$

and in Theorem 3 that if $2 \leq \gamma \leq 4$, then under a smoothness condition on the distribution of $C_{1}$,

$$
\varlimsup_{n} X_{n}=\frac{\gamma}{4} \text { w.p.1. }
$$


This leads to the interesting problem of determining the limit point set of the trajectories of $\left(X_{n}\right)_{0}^{\infty}$ in the critical case. We do not address this problem here and plan to study it in a sequel.

Athreya and Dai [2] have shown that in the critical case the occupation measures

$$
\mu_{n, x}(.)=\frac{1}{n} \sum_{0}^{n-1} P_{x}\left(X_{j} \in .\right)
$$

converge weakly to $\delta_{0}$, the delta measure at zero. This raises the question of what happens to the empirical measure

$$
V_{n}(.)=\frac{1}{n} \sum_{0}^{n-1} I\left(X_{j} \in .\right) .
$$

Using results from the theory of random walks we show in Theorem 4 that $V_{n}$ converges to $\delta_{0}$ weakly w.p.1 for any $P_{x}, x \in(0,1) \backslash \triangle$.

Our main result, i.e. Theorem 1 , has an interesting implication for ecological modelling. Here $X_{n}$ represents the population density in a given habitat during the $n$th generation normalized to take values in $[0,1]$. The random parameter $C_{n+1}$ represents the (stochastic) demographic growth rate in the period $n$ to $(n+1)$. In the subcritical case (i.e. $\left.E\left(\log C_{1}\right)<0\right)$ the stochasticity of this parameter is not enough to prevent the population density $X_{n}$ decaying geometrically fast w.p.1 as shown by Athreya and Dai $[2]$.

In the critical case the stochasticity of this parameter is enough to prevent the population from dying out. Thus even though for large $n$ the population density $X_{n}$ is small with a high probability the trajectory of $X_{n}$ rises to heights $\beta$ and beyond again and again w.p.1 for any initial value $X_{0}=x, x \in(0,1) \backslash \triangle$, and if 4 belongs to the support of $C_{1}$, it can even get close to the full value 1 infinitely often under some 
regularity conditions on $C_{1}$. This raises the question of how often do such "peaks" occur or equivalently what can can one say about the time interval between such "peaks"? We do not pursue this here.

After a section on some review of the literature and some preliminary results that will be used in the sequel and are also of independent interest the persistence of $\left(X_{n}\right)_{0}^{\infty}$ is discussed in section 3 where Theorem 1 is stated and proved. Section 4 deals with the maximum level that a trajectory can achieve, while the almost sure weak convergence of the empirical measure is treated in section 5 .

\section{$2 \quad$ Review and some preliminary results}

Let $\left(X_{n}\right)_{0}^{\infty}$ be a Markov chain defined by (2). Athreya and Dai [2] established the following results:

Theorem A: Let $\mu$ be an invariant probability measure for $\left(X_{n}\right)_{0}^{\infty}$ such that $\mu((0,1))=$ 1. Then $E\left(\log C_{1}\right)>0$ and equals $-\int \log (1-x) \mu(d x)$.

Corollary 1: If $E\left(\log C_{1}\right) \leq 0$ then $\mu=\delta_{0}$ is the only invariant measure for $\left(X_{n}\right)_{0}^{\infty}$ and for all $0 \leq x \leq 1$,

$$
\mu_{n, x} \rightarrow \delta_{0} \text { weakly }
$$

where $\mu_{n, x}(.) \equiv \frac{1}{n} \sum_{0}^{n-1} P_{x}\left(X_{j} \in.\right)$ is the occupation measure.

Theorem B: If $E\left(\log C_{1}\right)=0$ and $P\left(C_{1}=1\right)<1$, then

$$
X_{n} \rightarrow 0 \text { in probability. }
$$


The proof in [2] uses a comparison result (step 1 on page 603) which can be improved by employing the following Theorem $\mathrm{C}$, which may be also of independent interest.

Theorem C (comparison lemma): Let $\left(Y_{n}\right)_{0}^{\infty}$ be a sequence of random variables defined using the same sequence $\left(C_{n}\right)_{0}^{\infty}$ as in (2) but by the following random iteration scheme:

$$
Y_{n+1}=\min \left(C_{n+1} Y_{n}\left(1-Y_{n}\right), \frac{1}{2}\right)
$$

with $Y_{0}=\min \left(X_{0}, \frac{1}{2}\right)$.

Then for all $n \geq 0, Y_{n} \geq \min \left(X_{n}, \frac{1}{2}\right)$ w.p.1.

Proof: For $n=0$ equality holds by definition. By induction, assume true for $n$. Then

$$
\begin{aligned}
Y_{n+1} & =\min \left(C_{n+1} Y_{n}\left(1-Y_{n}\right), \frac{1}{2}\right) \geq \min \left(C_{n+1} \min \left(X_{n}, \frac{1}{2}\right)\left(1-\min \left(X_{n}, \frac{1}{2}\right)\right), \frac{1}{2}\right) \\
& \geq \min \left(C_{n+1} X_{n}\left(1-X_{n}\right), \frac{1}{2}\right)=\min \left(X_{n+1}, \frac{1}{2}\right) .
\end{aligned}
$$

since $g_{1}(x)=x(1-x)$ is monotone nondecreasing in $\left[0, \frac{1}{2}\right]$.

Corollary 2: For all $0<\epsilon \leq \frac{1}{2}, 0 \leq x \leq \frac{1}{2}$ and $n \geq 1$ :

$$
P_{x}\left(X_{n} \geq \epsilon\right)\left(=P_{x}\left(\min \left(X_{n}, \frac{1}{2}\right) \geq \epsilon\right)\right) \leq P_{x}\left(Y_{n} \geq \epsilon\right)
$$

Corollary 2 is step 1 in the proof of Theorem B given in Athreya and Dai [2]. It is interesting to note that Theorem $\mathrm{C}$ has the following implication for population control mechanisms in ecology. In the growth model (2) where for small values the growth is approximately linear and large values in one generation lead to low values in the next generation a constant trimming at level $\frac{1}{2}$ in each generation leads to a population that dominates the untrimmed population most of the time sample path wise. It is worth noting that Theorem $\mathrm{C}$ is valid in all three cases, i.e. it does not require any conditions on $E\left(\log C_{1}\right)$. 
We need a result ruling out the possibility of $X_{n}=0$ for some $1 \leq n<\infty$. This is so as we use the sequence $U_{n} \equiv-\log X_{n}, n \geq 0$, as a tool in the proof of Theorem 1 in the next section. It is also of independent interest.

\section{Theorem D:}

i) If $P\left(C_{1}=4\right)=0$ then $P_{x}\left(X_{n}=0\right.$ for some $\left.n \geq 1\right)=0$ for all $0<x<1$.

ii) If $P\left(C_{1}=4\right)>0$ then there exists a countable set $\triangle \subset(0,1)$ such that $P_{x}\left(X_{n}=0\right.$ for some $\left.n \geq 1\right)=0$ for all $x \in(0,1) \backslash \triangle$.

Proof: Let $g_{c}(x) \equiv c x(1-x), 0 \leq x \leq 1$ and $0 \leq c \leq 4$. The equation $g_{c}(x)=1$ has no solution in $[0,1]$, if $c<4$, and has a unique solution, i.e. $x=\frac{1}{2}$, when $c=4$.Also for $n \geq 2$ the events $A_{n}=\left(X_{n}=0, X_{n-1} \neq 0\right), A_{n}^{\prime}=\left(X_{n-1}=1\right)$ and $A_{n}^{\prime \prime}=\left(X_{n-2}=\frac{1}{2}, C_{n-1}=4\right)$ coincide w.p.1 for any $P_{x}$.

Since by definition of $\left(X_{n}\right)_{0}^{\infty}$ in $(2)$

$$
\begin{aligned}
& X_{n+1}=g_{c_{n+1}}\left(X_{n}\right)=C_{n+1} X_{n}\left(1-X_{n}\right) \text {, we have } \\
& \qquad P_{x}\left(A_{n}\right)=P_{x}\left(A_{n}^{\prime \prime}\right)=P_{x}\left(X_{n-2}=\frac{1}{2}\right) P\left(C_{n-1}=4\right)
\end{aligned}
$$

for $n \geq 2$ and

$$
P_{x}\left(A_{1}\right)=0 \text { for all } 0<x<1
$$

i) Thus $P_{x}\left(\bigcup_{n=1}^{\infty} A_{n}\right)=0,0<x<1$, if $P\left(C_{1}=4\right)=0$, proving (i) 
ii) Let $B=\left\{x \mid P\left(C_{1}=x\right)>0\right\}$. Then $B$ is at most countable, say,

$B=\left\{a_{1}, a_{2}, \ldots\right\}$

For $n \geq 1$ let

$$
\triangle_{n} \equiv\left\{x \mid 0<x<1 ; g_{a_{i_{1}}}\left(\ldots\left(g_{a_{i_{n}}}(x)\right) \ldots\right)=\frac{1}{2} \text { for some } i_{1}, \ldots, i_{n} \geq 1\right\}
$$

Since for all $a_{i_{1}}, \ldots, a_{i_{n}}, n \geq 1, g_{a_{i_{1}}}\left(\ldots\left(g_{a_{i_{n}}}(x)\right) \ldots\right)$ is a polynomial of degree $2^{n}$, the set of $x$ for which this polynomial equals $\frac{1}{2}$ is finite and hence $\triangle_{n}$ is at most countable. Let

$$
\triangle \equiv\left(\bigcup_{1}^{\infty} \triangle_{n}\right) \cup\left\{\frac{1}{2}\right\}
$$

Then $\triangle$ is countable. Again using (3) we see that

$P_{x}\left(A_{2}\right)=P_{x}\left(A_{2}^{\prime \prime}\right)=P_{x}\left(X_{0}=\frac{1}{2}\right) P\left(C_{1}=4\right)>0 \Longrightarrow x=\frac{1}{2}$

and $P_{x}\left(A_{n}^{\prime \prime}\right)>0$ for $n>2$

$g_{a_{i_{1}}}\left(\ldots\left(g_{a_{i_{n-2}}}(x)\right) \ldots\right)=\frac{1}{2}$ for some $i_{1}, i_{2}, \ldots, i_{n-2} \geq 1$, i.e. $x \in \triangle_{n-2}$.

Thus $P_{x}\left(\bigcup_{1}^{\infty} A_{n}\right)>0 \Longrightarrow x \in \triangle$ proving (ii).

Remark 1: In the rest of the paper it will be assumed that the initial value $x$ will

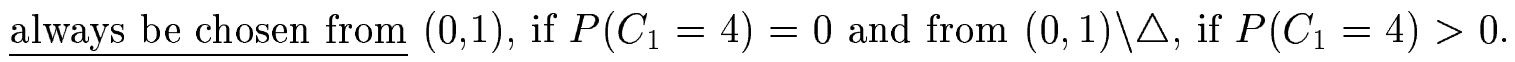
For $X_{0}$ random it will be assumed that $P\left(0<X_{0}<1\right)=1$ if $P\left(C_{1}=4\right)=0$ and $P\left(X_{0} \in(0,1) \backslash \triangle\right)=1$ if $P\left(C_{1}=4\right)>0$.

\section{$3 \quad$ Persistence of the critical logistic process}

Let $\left(X_{n}\right)_{0}^{\infty}$ be a Markov chain as in (2), i.e.

$$
X_{n+1}=g_{c_{n+1}}\left(X_{n}\right)=C_{n+1} X_{n}\left(1-X_{n}\right), \quad n \geq 0
$$

where $\left(C_{n}\right)_{0}^{\infty}$ are i.i.d. random variables with values in $(0,4]$ and independent of $X_{0}$ with values in $[0,1]$. The goal of this section is to prove Theorem 1 below which asserts 
that the critical logistic process is persistent, i.e. it keeps crossing a level $\beta>0$ again and again.

Theorem 1: If $E\left(\log C_{1}\right)=0$ and $P\left(C_{1}=1\right)<1$ then there exists a level $\beta, 0<\beta<1$, such that

$$
P_{x}\left(X_{n} \geq \beta \text { for infinitely many } n \geq 1\right)=1
$$

for any $0<x<1$, if $P\left(C_{1}=4\right)=0$, and for any $x \in(0,1) \backslash \triangle$, if $P\left(C_{1}=4\right)>0$, where $P_{x}$ stands for the probability distribution of $\left(X_{n}\right)_{0}^{\infty}$ conditioned on $X_{0}=x$ w.p. 1 and $\triangle$ is the countable set defined in (4).

Proof: Since $\left(X_{n}\right)_{0}^{\infty}$ is a Markov chain it suffices to show that there exists a $0<\beta<1$ such that

$$
P_{x}\left(X_{n} \geq \beta \text { for some } n \geq 1\right)=1
$$

for all $0<x<1,(x \notin \triangle)$.

We now claim that for any $\epsilon>0$ and $0<x<1,(x \notin \triangle)$,

$$
P_{x}\left(0<X_{n} \leq \epsilon \text { for some } n \geq 1\right)=1 \text {. }
$$

To see this note that

i) $X_{n} \leq C_{n} C_{n-1} \ldots C_{1}$

ii) $E\left(\log C_{1}\right)=0 \Longrightarrow \alpha=P\left(C_{1}<\rho\right)>0$ for some $\rho<1$

iii) $\inf _{0<x<1} P_{x}\left(X_{k} \leq \epsilon\right) \geq \alpha^{k}>0$, if $\rho^{k}<\epsilon$, and use the Markov property of $\left(X_{n}\right)_{0}^{\infty}$. 
Thus it suffices to establish (6) for $x \leq \epsilon, \quad \epsilon$ small.

Now if we let

$$
U_{n}=-\log X_{n}
$$

then it is enough to show that there exists a $0<K<\infty$ such that

$$
P_{u}\left(U_{n} \leq K \text { for some } n \geq 1\right)=1
$$

for all $u$ sufficiently large $\left(e^{-u} \notin \triangle\right)$. Note that by Theorem D and Remark $1, U_{n}$ is well defined. Taking logarithms on both sides of (2) yields

$$
U_{n+1}=D_{n+1}+U_{n}-\log \left(1-e^{-U_{n}}\right), n \geq 0,
$$

where $D_{n}=-\log C_{n}, n \geq 1$.

Let $K>\log 4+1$ and

$$
\begin{aligned}
& T=\inf \left\{n \mid n \geq 1, U_{n}<K\right\}(\inf \phi:=\infty), \\
& Z_{n}=\log U_{n \wedge T}, n \geq 0, \text { with } Z_{0}=\log u .
\end{aligned}
$$

Since $\log C_{1} \leq \log 4$ w.p.1, $U_{n} \geq K$ for $n<T$ and $U_{T} \geq K-\ln 4$, if $T<\infty$, it follows that $Z_{n} \geq \log (K-\log 4)>\log 1=0$.

We now claim that there exists a large $K$ such that

$$
\left(Z_{n}\right)_{0}^{\infty} \text { is a supermartingale. }
$$

Assume for the moment that (13) holds. Then since $\left(Z_{n}\right)_{0}^{\infty}$ is a nonnegative supermartingale it converges w.p.1. If $Z_{\infty} \equiv \lim _{n} Z_{n}$, then $\log (K-\log 4) \leq Z_{\infty}<\infty$. Let 
$B_{1}=\left(\lim _{n} Z_{n} \equiv Z_{\infty}\right.$ exists $), B_{2}=(T=\infty)$ and $B_{3}=\left(\lim _{n} D_{n}\right.$ exists $)$. On the event $B_{1} \cap B_{2}, U_{n} \rightarrow U_{\infty} \in[K-\log 4, \infty)$ and hence by $(9), \lim _{n} D_{n}$ exists. Thus $B_{1} \cap B_{2} \subset B_{3}$. But since $\left(D_{n}\right)_{1}^{\infty}$ are i.i.d. nondegenerate random variables, $P\left(B_{3}\right)=0$. As noted above, (13) implies that $P_{u}\left(B_{1}\right)=1$. Thus $P_{u}\left(B_{2}\right)=P_{u}\left(B_{1} \cap B_{2}\right) \leq P\left(B_{3}\right)=0$. Therefore $P_{u}(T<\infty)=1$ and hence (8) holds.

It remains only to establish (13). As $\left(Z_{n}\right)_{0}^{\infty}$ is a Markov chain, it is enough to show that for $K$ large and $u>K-\log 4, e^{-u} \notin \triangle$,

$$
E\left(Z_{n+1} \mid Z_{n}=\log u\right) \leq \log u
$$

If $u<K$, then $T \leq n$, and hence $Z_{n}=\log u \Longrightarrow Z_{n+1}=\log u$ and so (14) holds. If $u \geq K$, using (9) we get

$$
\begin{gathered}
\qquad h(u) \equiv E\left(Z_{n+1} \mid Z_{n}=\log u\right)-\log u= \\
=E\left(\log \left(u+D+\left(-\log \left(1-e^{-u}\right)\right)-\log u\right)\right. \\
\text { where } D \text { is a random variable with distribution } D_{1}=-\log C_{1} \\
=E\left(\log \left(1+\frac{D+a(u)}{u}\right)\right), \text { where } a(u)=-\log \left(1-e^{-u}\right) .
\end{gathered}
$$

Also, since $E(D)=0$,

$h(u)=E\left(\log \left(1+\frac{D+a(u)}{u}\right)-\frac{D}{u}\right)$.

Now, (14) is equivalent to

$$
h(u) \leq 0 \text { for } u \text { large, }\left(e^{-u} \notin \triangle\right)
$$

Let $L>0$ be such that $P(D \leq L)>0$. Since $a(u)=o\left(\frac{1}{u^{k}}\right)$ as $u \rightarrow \infty$ for all $k \leq 1$ and 


$$
\begin{aligned}
\log (1+\epsilon) & =\epsilon-\frac{\epsilon^{2}}{2}+o\left(\epsilon^{2}\right) \text { as } \epsilon \rightarrow 0 \\
h_{1}(u) & \equiv E\left(\log \left(1+\frac{D+a(u)}{u}\right)-\frac{D}{u} ; D>L\right)= \\
& =E\left(\log \left(\left(1+\frac{D}{u}\right) e^{-\frac{D}{u}}+\frac{a(u)}{u} e^{-\frac{D}{u}}\right) ; D>L\right) \\
& \leq E\left(\log \left(1+\frac{a(u)}{u}\right) ; D>L\right), \text { using } 1+y \leq e^{y} \text { for } y>0 \\
& \leq \log \left(1+\frac{a(u)}{u}\right)=o\left(\frac{1}{u^{2}}\right) \text { as } u \rightarrow \infty
\end{aligned}
$$

Also

$$
\begin{aligned}
h_{2}(u) & \equiv E\left(\log \left(1+\frac{D+a(u)}{u}\right)-\frac{D}{u} ; D \leq L\right)= \\
& =E\left(\frac{D+a(u)}{u}-\frac{1}{2}\left(\frac{D+a(u)}{u}\right)^{2}+o\left(\frac{1}{u^{2}}\right)-\frac{D}{u} ; D \leq L\right)= \\
& =-\frac{1}{2} E\left(\left(\frac{D+a(u)}{u}\right)^{2} ; D \leq L\right)+o\left(\frac{1}{u^{2}}\right)= \\
& =-\frac{1}{2} \frac{E\left(D^{2} ; D \leq L\right)}{u^{2}}+o\left(\frac{1}{u^{2}}\right) .
\end{aligned}
$$

Since $P(D \leq L)>0, \quad E\left(D^{2} ; D \leq L\right)>0$.

Thus $\varlimsup_{u \rightarrow \infty} u^{2} h(u) \leq-\frac{1}{2} E\left(D^{2} ; D \leq L\right)<0$,

implying that there exists $u_{0}$ so large that $h(u)<0$ for $u \geq u_{0}$, establishing (16).

Now choose $K>u_{0}+\log 4+1$.

\section{The $\varlimsup$ im of the sample path in the critical case}

¿From Theorem 1 we note that the process $\left(X_{n}\right)_{0}^{\infty}$ goes above a level $\beta, 0<\beta<1$, infinitely often. It is intuitive that starting from a value $x_{0} \geq \beta$ the sequence $\left(X_{n}\right)_{0}^{\infty}$ could have a positive probability of going higher before going down below $\beta$. The goal of this section is to explore the $\varlimsup$ im of the sequence $\left(X_{n}\right)_{0}^{\infty}$. The assumption about $x$ mentioned in Remark 1 will be in force throughout this section.

\section{Definition:}




$$
\gamma \equiv \sup \left\{x \mid P\left(C_{1}<x\right)<1\right\}
$$

be the supremum of the support of the distribution of $C_{1}$.

Theorem 2: Let $E\left(\log C_{1}\right)=0, P\left(C_{1}=1\right)<1$.

i) If $1<\gamma \leq 2$, i.e. $P\left(C_{1} \leq 2\right)=1$, then $P_{x}\left(\varlimsup_{n} X_{n}=1-\frac{1}{\gamma}\right)=1$.

ii) If $2<\gamma \leq 4$, i.e. $P\left(C_{1} \leq 2\right)<1$, then $P_{x}\left(\varlimsup_{n} X_{n} \geq 1-\frac{1}{\gamma}\right)=1$.

\section{Proof:}

i) If $\gamma \leq 2$ then w.p.1,

$X_{n}=C_{n} X_{n-1}\left(1-X_{n-1}\right) \leq \frac{C_{n}}{4} \leq \frac{\gamma}{4} \leq \frac{1}{2}$ for all $n \geq 1$.

So we may assume without loss of generality (w.l.o.g.) that $X_{0} \leq \frac{1}{2}$.

Also $g_{c}(x)=c x(1-x)$ is nondecreasing in $x$ for $x \in\left[0, \frac{1}{2}\right]$ and nondecreasing in $c$ for $c \in(0,4]$.

Thus for $n \geq 1, X_{n+1}=g_{c_{n+1}}\left(X_{n}\right) \leq g_{\gamma}\left(X_{n}\right)$ w.p.1.

Since $X_{n} \leq \frac{1}{2}$ for $n \geq 0$ and $g_{\gamma}($.$) is nondecreasing in \left[0, \frac{1}{2}\right]$ we get by iteration that $X_{n} \geq g_{\gamma}^{(n)}\left(X_{0}\right)$.

But $g_{\gamma}^{(n)}(x) \rightarrow 1-\frac{1}{\gamma}$ for all $0<x<1$. So

$$
\varlimsup_{n} X_{n} \leq 1-\frac{1}{\gamma} \text { w.p.1. }
$$

Choose $\beta$ (of Theorem 1$)<\frac{1}{2}$. For any $0<\eta<\gamma$,

$$
\alpha \equiv P\left(\eta \leq C_{1} \leq \gamma\right)>0
$$

Also for $1<c<3, g_{c}^{(n)}(x) \rightarrow 1-\frac{1}{c}$ uniformly for $x \in\left[\beta, \frac{1}{2}\right]$. Now let $C_{1}, C_{2}, \ldots, C_{n}$ 
$\in[\eta, \gamma]$. By monotonicity of $c \longmapsto g_{c}(),. g_{\eta}\left(X_{0}\right) \leq X_{1}\left(=g_{c_{1}}\left(X_{0}\right)\right) \leq g_{\gamma}\left(X_{0}\right)$

and hence $g_{\eta}^{(n)}\left(X_{0}\right) \leq X_{n} \leq g_{\gamma}^{(n)}\left(X_{0}\right)$ for $n \geq 1$ by induction.

But $g_{\eta}^{(n)}(x) \rightarrow 1-\frac{1}{\eta}$ for $0<x<1$. So for $\epsilon>0$ there exists $n_{0}$ such that $g_{\eta}^{\left(n_{0}\right)}(\beta)>\left(1-\frac{1}{\eta}\right)-\epsilon$ and hence

$$
g_{\eta}^{\left(n_{0}\right)}>\left(1-\frac{1}{\eta}\right)-\epsilon \text { for all } \beta \leq x \leq \frac{1}{2} .
$$

Consider the stopping times defined by

$$
\begin{aligned}
& T_{1}=\inf \left\{n \mid n>0 X_{n}<\beta\right\}, \\
& T_{2}=\inf \left\{n \mid n>T_{1}, X_{n} \geq \beta\right\} \text { and recursively } \\
& T_{2 k+1}=\inf \left\{n \mid n>T_{2 k}, X_{n}<\beta\right\}, k \geq 0 \\
& T_{2 k}=\inf \left\{n \mid n>T_{2 k-1}, X_{n} \geq \beta\right\}, k \geq 1 .
\end{aligned}
$$

Now, Theorem 1 and Theorem B imply that

$P_{x}\left(T_{n}<\infty\right.$ for all $\left.n \geq 1\right)=1$.

Let $A_{k}=\left(X_{n} \geq\left(1-\frac{1}{\eta}\right)-\epsilon\right.$ for some $\left.n \in\left[T_{2 k}, T_{2 k+1}\right)\right)$.

Then by the strong Markov property,

$P\left(A_{k} \mid X_{T_{2 k}}\right) \geq P\left(C_{j} \in[\eta, \gamma], j=1,2, \ldots, n_{0}\right) \geq \alpha^{n_{0}}$

where $\alpha$ and $n_{0}$ are as in (19) and (20).

Therefore by the conditional Borel-Cantelli lemma (Durrett [8], page 240)

$P\left(A_{k}\right.$ infinitely often $)=1$. So $\varlimsup_{n} X_{n} \geq(1-\eta)-\epsilon$ w.p.1.

This being true for every $\eta<\gamma$ and $\epsilon>0$, it follows that $\overline{\lim } X_{n} \geq 1-\gamma$ w.p.1 and we are done in view of (18).

ii) It is known (Devaney [7]) that

$g_{c}^{(n)}(x) \rightarrow 1-\frac{1}{c}$, if $1 \leq c \leq 3$ and $0<x<1$, and it can be shown that 
$\varlimsup_{n} g_{c}^{(n)}(x) \geq 1-\frac{1}{c}$, if $3<c<4$ and $0<x<1$.

Also, $\varlimsup_{n} g_{4}^{(n)}(x)=1$ for almost all $0<x<1$.

Thus, if $c$ is in the support of the distribution of $C_{1}$, proceeding as in the proof of (i) one shows that $\varlimsup_{n} X_{n} \geq 1-\frac{1}{c}$ w.p.1 and hence (ii) follows.

Remark 2: If for $\gamma>3, g_{\gamma}(x)$ has a cyclic orbit with largest value $A$, then (ii) of Theorem 2 can be strengthened to $P_{x}\left(\varlimsup_{n} X_{n} \geq A\right)=1$.

The next result identifies the $\varlimsup_{n} X_{n}$ when $2 \leq \gamma \leq 4$ improving (ii) of Theorem 2 . It requires some smoothness conditions on the distribution of $C_{1}$. We do not believe that they are necessary.

Theorem 3: Let $E\left(\log C_{1}\right)=0, P\left(C_{1}=1\right)<1$.

Let $\gamma>2$, i.e. $P\left(C_{1}>2\right)>0$. Suppose the distribution of $C_{1}$ satisfies either one of the following two conditions:

A) There is $d>1$ such that for each $\epsilon>0$

$$
\inf \left\{P\left(a-\epsilon<C_{1}<a+\epsilon\right) \mid \quad \frac{1}{\gamma} \leq a \leq d\right\}>0
$$

B) For each $\epsilon>0$

$$
\inf \left\{P\left(a-\epsilon<C_{1}<a+\epsilon\right) \mid \quad 1 \leq a \leq \gamma\right\}>0
$$

Then $P_{x}\left(\varlimsup_{n} X_{n}=\frac{\gamma}{4}\right)=1$.

Proof: Fix $0<\theta<1$ and $0<\eta<\gamma$. Let $B=\left(X_{j} \geq \frac{\theta \cdot \eta}{4}\right.$ for some $\left.j \geq 1\right)$. 
Suppose it is shown that there exist a sequence of stopping times $\left(\tau_{j}\right)_{1}^{\infty}, P_{x}\left(\tau_{j}<\infty\right)=$ 1 for all $j$, and $0<\lambda<\infty$ such that

$$
P_{X_{\tau_{j}}}(B) \geq \lambda \text { w.p.1, }
$$

then, by the conditional Borel-Cantelli lemma (Durrett [8], page 240), $P_{x}\left(X_{j} \geq \frac{\theta \cdot \eta}{4}\right.$ for infinitely many $\left.j\right)=1$ and so $P_{x}\left(\varlimsup_{n} X_{n} \geq \frac{\theta \cdot \eta}{4}\right)=1$.

This being true for all $0<\theta<1$ and $0<\eta<\gamma$ it follows that $P_{x}\left(\overline{\lim _{n}} X_{n} \geq \frac{\gamma}{4}\right)=1$. On the other hand, for $n \geq 1, X_{n} \leq \frac{C_{n}}{4} \leq \frac{\gamma}{4}$ w.p.1 and hence $P_{x}\left(\varlimsup_{n} X_{n} \leq \frac{\gamma}{4}\right)=1$. It remains only to establish (22). In order that $X_{n+1}>\frac{\theta}{4} \cdot \eta$, it suffices that $\left|X_{n}-\frac{1}{2}\right|<\delta$ and $C_{n+1}>\eta$, where $\delta>0$ is such that $g_{1}(x) \equiv x(1-x) \geq \frac{\theta}{4}$ for $\left|x-\frac{1}{2}\right|<\delta$.

Thus $P_{x}\left(X_{n+1}>\frac{\theta \cdot \eta}{4}\right) \geq P_{x}\left(\left|X_{n}-\frac{1}{2}\right|<\delta\right) \cdot P\left(\eta \leq C_{n+1} \leq \gamma\right)$.

Hence if it is shown that for each $\delta>0$ there exists a sequence of stopping times $\left(\tau_{j}\right)_{1}^{\infty}, P_{x}\left(\tau_{j}<\infty\right)=1$ for all $j \geq 1$, an $n_{0} \geq 1$ and a $\tilde{\lambda}>0$ such that

$$
P_{X_{\tau_{j}}}\left(\left|X_{n_{0}}-\frac{1}{2}\right|<\delta\right) \geq \tilde{\lambda} \text { for } j \geq 1
$$

then (22) follows.

The function $g_{\gamma}(x)=\gamma x(1-x)$ is strictly increasing on $\left[0, \frac{1}{2}\right]$ with range $\left[0, \frac{\gamma}{4}\right]$, which includes $\left[0, \frac{1}{2}\right]$ if $\gamma>2$. Thus $g_{\gamma}^{-1}$ is well defined on $\left[0, \frac{1}{2}\right]$ and its range is contained in $\left[0, \frac{1}{2}\right]$. Hence $\left(g_{\gamma}^{-1}\right)^{(k)}$ is well defined for all $k \geq 1$.

Let $x_{1}=g_{\gamma}^{-1}\left(\frac{1}{2}\right)$ and $x_{k+1}=g_{\gamma}^{-1}\left(x_{k}\right)$ for $k \geq 1$. Then $x_{k} \downarrow 0$. Now choose $k_{0}$ so large, that $\beta=x_{k_{0}}$ can serve as a level achieved in Theorem 1. W.l.o.g. let $\beta<\frac{1}{8}$, i.e. $4 \beta<\frac{1}{2}$, and $\frac{1}{1-\beta} \leq d$, where $d$ is as in condition (A).

Since $g_{c}^{k_{0}}(x)$ is continuous in $[0,1] \mathbf{x}(0,4]$ as a function of $(x, c)$ and since $g_{\gamma}^{\left(k_{0}\right)}(\beta)=\frac{1}{2}$ for any $\delta>0$, there exist $h_{1}>0, h_{2}>0$ such that

$$
\left|g_{c}^{\left(k_{0}\right)}(x)-\frac{1}{2}\right|<\delta, \text { if }|x-\beta|<h_{1} \text { and }|c-\gamma|<h_{2}
$$


Thus, if $C_{1}, C_{2}, \ldots, C_{k_{0}} \in(\eta, \gamma], \eta:=\gamma-h_{2}$, and $|x-\beta|<h_{1}$, then

$$
X_{k_{0}} \equiv g_{C_{k_{0}}}\left(\ldots\left(g_{C_{1}}(x)\right) \ldots\right) \in\left(\frac{1}{2}-\delta, \frac{1}{2}+\delta\right)
$$

and hence

$P_{x}\left(X_{k_{0}} \in\left(\frac{1}{2}-\delta, \frac{1}{2}+\delta\right)\right) \geq\left(P\left(\eta<C_{1} \leq \gamma\right)\right)^{k_{0}} \equiv \alpha^{k_{0}}>0$.

To establish (23) it suffices to find stopping times $\left(\tau_{j}\right)_{1}^{\infty}, P_{x}\left(\tau_{j}<\infty\right)=1$ for all $j \geq 1$, and a $\widetilde{\widetilde{\lambda}}>0$ such that

$$
P_{X_{\tau_{j}}}\left(\left|X_{1}-\beta\right|<h_{1}\right)>\widetilde{\widetilde{\lambda}} \text { w.p. } 1 \text { for all } j \geq 1
$$

since then

$$
P_{X_{\tau_{j}}}\left(\left|X_{k_{0}+1}-\frac{1}{2}\right|<\delta\right) \geq \widetilde{\widetilde{\lambda}} \alpha^{k_{0}}>0
$$

By Theorem 1 and Theorem B we know that $\left(X_{n}\right)_{0}^{\infty}$ crosses $\beta$ from both sides infinitely often. Let $\left(T_{n}\right)_{0}^{\infty}$ be the stopping times defined as in (21), i.e.

$$
\begin{aligned}
& T_{0} \quad \equiv 0 ; T_{1}=\inf \left\{n \mid n>0, X_{n}<\beta\right\} \\
& T_{2} \quad=\inf \left\{n \mid n>T_{1}, X_{n} \geq \beta\right\} \text { and recursively, } \\
& T_{2 k+1}=\inf \left\{n \mid n>T_{2 k}, X_{n}<\beta\right\} \text { for } k \geq 0 \\
& T_{2 k} \quad=\inf \left\{n \mid n>T_{2 k-1}, X_{n} \geq \beta\right\} \text { for } k \geq 1
\end{aligned}
$$

Then by Theorem 1 and Theorem B

$$
P_{x}\left(T_{n}<\infty \text { for all } n\right)=1 \text {. }
$$

Since $\beta \leq X_{T_{2 k}} \leq \gamma \beta<\frac{1}{2}$ w.p.1,

$$
\begin{aligned}
& P_{X_{T_{2 k}}}\left(\left|X_{1}-\beta\right|<h_{1}\right) \geq \inf _{\beta \leq y \leq \gamma \beta} P\left(\frac{\beta}{y(1-y)}-\frac{h_{1}}{y(1-y)}<C_{1}<\frac{\beta}{y(1-y)}+\frac{h_{1}}{y(1-y)}\right) \\
\geq & \inf \left\{P\left(a-\delta^{\prime}<C_{1}<a+\delta^{\prime}\right) \mid \quad \frac{\beta}{\gamma \beta(1-\gamma \beta)} \leq a \leq \frac{\beta}{\beta(1-\beta)}\right\} \\
& \text { where } \delta^{\prime}=\frac{h_{1}}{\gamma \beta(1-\gamma \beta)} \\
\geq & \inf \left\{P\left(a-\delta^{\prime}<C_{1}<a+\delta^{\prime}\right) \mid \quad \frac{1}{\gamma} \leq a \leq \frac{1}{1-\beta}\right\} .
\end{aligned}
$$

Since $\frac{1}{1-\beta} \leq d$, condition (A) now implies (24) for the sequence $\tau_{j}=T_{2 j}, j \geq 1$. 
Let $\left(T_{n}^{\prime}\right)_{0}^{\infty}$ be the stopping times defined as in (21) but with $\beta$ replaced by $\widetilde{\beta}=x_{k_{0}+1}=$ $g_{\gamma}^{-1}(\beta)$. Hence

$$
\begin{aligned}
& \tilde{\beta}=g_{\gamma}^{-1}(\beta) \leq X_{T_{2 k}^{\prime}} \leq \beta . \\
& P_{X_{T_{2 k}}}\left(\left|X_{1}-\beta\right|<h_{1}\right)=\inf _{\widetilde{\beta} \leq y \leq \beta} P\left(\frac{\beta}{y(1-y)}-\frac{h_{1}}{y(1-y)}<C_{1}<\frac{\beta}{y(1-y)}+\frac{h_{1}}{y(1-y)}\right) \\
& \geq \inf \left\{P\left(a-\delta^{\prime \prime}<C_{1}<a+\delta^{\prime \prime}\right): \frac{\beta}{\beta(1-\beta)} \leq a \leq \min \left(\frac{b}{b(1-b)}, \gamma\right)\right\}, \text { where } \delta^{\prime \prime}=\frac{h_{1}}{\beta(1-\beta)}, \\
& \left.\geq \inf \left\{P\left(a-\delta^{\prime \prime}<C_{1}<a+\delta^{\prime \prime}\right): \frac{1}{1-\beta} \leq a \leq \gamma\right)\right\} \\
& \left.\geq \inf \left\{P\left(a-\delta^{\prime \prime}<C_{1}<a+\delta^{\prime \prime}\right): 1 \leq a \leq \gamma\right)\right\} .
\end{aligned}
$$

If (B) holds then (24) holds for the sequence $\tau_{j}=T_{2 j}^{\prime}, j \geq 1$, and the proof is complete.

Remark 3: If the distribution of $C_{1}$ has an absolutely continuous component with density $f$, then (A) holds, if for some $d>1, \inf _{\frac{1}{\gamma} \leq x \leq d} f(x)>0$, and (B) holds, if $\inf _{1 \leq x \leq \gamma} f(x)>0$.

\section{$5 \quad$ Almost sure convergence of the empirical measure}

For the critical case Athreya and Dai [2] have shown that the occupation measure $\mu_{n, x}(A) \equiv \frac{1}{n} \sum_{0}^{n-1} P_{x}\left(X_{j} \in A\right)$ converges weakly to $\delta_{0}$, the delta measure at 0 . Now $\mu_{n, x}(A)=E_{x}\left(V_{n}(A)\right)$, where $V_{n}$ is the empirical measure. The goal of this section is to show that w.p.1, $V_{n}$ converges weakly to $\delta_{0}$.

Theorem 4: If $E\left(\log C_{1}\right)=0$, then the empirical measure $V_{n}$ converges weakly to $\delta_{0}$ with probability one for any initial distribution of $X_{0}$ where 


$$
V(A):=\frac{1}{n} \sum_{0}^{n-1} I\left(X_{j} \in A\right) \text { for Borel sets } A \subset[0,1] .
$$

Proof: If $C_{1}=1$ w.p. 1 then $X_{n} \rightarrow 0$ w.p.1 as $n \rightarrow \infty$. Hence we assume that $P\left(C_{1}=1\right)<1$. We shall show that for every level $\beta>0$

$$
V_{n}([0, \beta]) \rightarrow 1 \text { w.p.1 as } n \rightarrow \infty \text {. }
$$

Consider the Markov chain $\left(U_{n}\right)_{0}^{\infty}$ with $U_{n}:=-\log X_{n}$.

Clearly, (25) is equivalent to

$$
\widetilde{V_{n}}([K, \infty)) \rightarrow, 1 \text { w.p.1 as } n \rightarrow \infty
$$

where $\widetilde{V}_{n}(A):=\frac{1}{n} \sum_{0}^{n-1} I\left(U_{j} \in A\right)$ for Borel sets $A \subset(0, \infty)$.

By Theorem 1, w.l.o.g., we may assume that $K>0$ is so large that $U_{n}<K$ infinitely often w.p.1. Recall from section 3 equation (9) that $\left(U_{n}\right)_{0}^{\infty}$ satisfies the recursion $U_{n+1}=D_{n+1}+U_{n}-\log \left(1-e^{-U_{n}}\right)$, where $D_{n}=-\log C_{n}$ are i.i.d., $E\left(D_{1}\right)=0$ and $D_{1} \not \equiv 0$.

Assume that $U_{0} \geq K$. The case $U_{0}<K$ is treated analogously.

Define the stopping times $T_{0} \equiv 0$,

$$
\begin{aligned}
& T_{2 k+1}:=\inf \left\{n \mid n>T_{2 k} \text { and } U_{n}<K\right\}, k=0,1, \ldots, \\
& T_{2 k}: \quad=\inf \left\{n \mid n>T_{2 k-1} \text { and } U_{n} \geq K\right\}, k=1,2, \ldots,
\end{aligned}
$$

and the random variables $\eta_{k}:=T_{2 k-1}-T_{2 k-2}$ and $\xi_{k}:=T_{2 k}-T_{2 k-1}, k=1,2,3, \ldots$

Now let $\eta:=-\log \left(1-e^{-K}\right)>0$.

Consider the two random walks:

$R_{n}:=\sum_{j=1}^{n} D_{j}$ and $S_{n}:=\sum_{j=1}^{n}\left(D_{j}+\eta\right), n=0,1,2, \ldots$, 
and the stopping times $\rho:=\inf \left\{n \mid R_{n}<0\right\}$ and $\sigma:=\inf \left\{n \mid S_{n} \geq K\right\}$. Also $E\left(D_{1}\right)=0$ and $E\left(D_{1}+\eta\right)=\eta>0$. Truncating $D_{n}$, Wald's equation (see e.g. Durrett [8]) implies that

$$
E(\rho)=\infty \text { and } E(\sigma)<\infty .
$$

¿From (9) we see that $\left(R_{n}\right)$ is stochastically smaller than $\left(U_{n}\right)$ on $\left[T_{2 k}, T_{2 k+1}\right]$ and $\left(S_{n}\right)$ is stochastically smaller than $\left(U_{n}\right)$ on $\left[T_{2 k+1}, T_{2 k+2}\right]$. As $\left(U_{n}\right)_{0}^{\infty}$ is a Markov chain, there exist i.i.d. copies $\left(\rho_{n}\right)_{n \geq 1}$ of $\rho$ and i.i.d. copies $\left(\sigma_{n}\right)_{n \geq 1}$ of $\sigma$, which are independent of each other, such that

$$
\eta_{j} \geq \rho_{j} \text { and } \xi_{j} \leq \sigma_{j}, j=1,2, \ldots
$$

Now define for $n \in\left[T_{2 k}, T_{2 k+1}\right]$

$N_{\eta}(n)=N_{\xi}(n)=k, \widetilde{\eta}(n)=n-\sum_{j=1}^{k} \eta_{j}-\sum_{j=1}^{k} \xi_{j}, \widetilde{\xi}(n)=0$,

and for $n \in\left[T_{2 k+1}, T_{2 k+2}\right]$

$N_{\eta}(n)=k+1$ and $N_{\xi}(n)=k, \widetilde{\eta}(n)=0, \widetilde{\xi}(n)=n-\sum_{j=1}^{k+1} \eta_{j}-\sum_{j=1}^{k} \xi_{j}, k=0,1,2, \ldots$,

(28) and (27) imply that

$$
\begin{aligned}
1 & \geq \widetilde{V}([K, \infty))=\frac{1}{n} \sum_{j=0}^{n-1} I\left(U_{j} \geq K\right)=\frac{1}{n}\left(\sum_{k=1}^{N_{n}(n-1)} \eta_{k}+\widetilde{\eta}(n-1)+1\right)= \\
& =\left(\sum_{k=1}^{N_{\eta}(n-1)} \eta_{k}+\widetilde{\eta}(n-1)+1\right) /\left(\sum_{k=1}^{N_{\eta}(n-1)} \eta_{k}+\widetilde{\eta}(n-1)+\sum_{k=1}^{N_{\xi}(n-1)} \xi_{k}+\widetilde{\xi}(n-1)+1\right)= \\
& =\left(1+\left(\sum_{k=1}^{N_{\xi}(n-1)} \xi_{k}+\widetilde{\xi}(n-1)\right) /\left(\sum_{k=1}^{N_{\eta}(n-1)} \eta_{k}+\widetilde{\eta}(n-1)+1\right)\right)^{-1} \geq \\
& \geq\left(1+\left(\sum_{k=1}^{N_{\xi}(n-1)+1} \sigma_{k}\right) /\left(\sum_{k=1}^{N_{\eta}(n-1)} \rho_{k}\right)\right)^{-1} \text { w.p.1 as } n \rightarrow \infty .
\end{aligned}
$$

by the strong law of large numbers, as $\left|N_{\eta}(n-1)-N_{\xi}(n-1)\right| \leq 1$.

Hence (26) is established.

Acknowledgement: The research reported in this paper was done while the second 
author was on a visit to the School of ORIE, Cornell University, whose hospitality and support is gratefully acknowledged.

\section{References:}

1. Athreya, K. B. and Bhattacharya, R. N. (2000). Random Iterations of I.I.D. Quadratic Maps. In "Stochastics in Finite and Infinite Dimensions" in honor of G. Kallianpur, 49-58, Birkhäuser.

2. Athreya, K. B. and Dai, Y. (2000). Random Logistic Maps I. J. Theor. Prob. $13,595-608$.

3. Bhattacharya, R. N. and Majumdar, M. (1999). On a theorem of Dubins and Freedman, J. Theor. Prob. 12, 1165-1185.

4. Bhattacharya, R. N. and Rao, B. V. (1993). Random Iterations of two quadratic maps. In Cambanis et al. (eds.), Stochastic Processes: A Festschrift in Honor of G. Kallianpur, Springer-Verlag.

5. Bhattacharya, R. N. and Waymire, E. C. (1990). Stochastic Processes with Applications, Wiley, New York.

6. Dai, J. (2000). A result regarding convergence of random logistic maps, Stat. and Prob. Letters 47, 11-14.

7. Devaney, R. L. (1989). An Introduction to Chaotic Dynamical Systems, 2nd Ed., Addison-Wesley, New York.

8. Durrett, R. (1996). Probability: Theory and Examples, Wadsworth, Pacific Grove, California.

9. May, R. H. (1976). Simple mathematical models with very complicated dynamics. Nature 261, 459-467. 